\title{
De la iglesia como baluarte al baluarte como iglesia. Estrategias de fortificación y modernización de las defensas medievales en las villas del litoral de la Marina de la costa de Alicante (1535-1587)
}

\section{Antoni Banyuls Pérez}

Universidad de Alicante, Alicante, España, antonibanyuls@gmail.com

\begin{abstract}
In the sixteenth century the coast between Calpe and Cape San Antonio in Jávea became the most dangerous in the Valencian coast against attacks by Barbary due to its exposed geographical location and rugged morphology. In the Middle Ages a system of defensive coastal villas protecting the territory of the incursions is configured, in this area of La Marina. This fact affected the development of an elementary urban scheme where the church-bastions constituted practically the only public building and therefore multifunctional. In the Renaissance, these structures were outdated address new strategies of fortification which led to these villas have to adapt to the new situation; taking into account also the new context of population growth and urban sprawl.
\end{abstract}

Keywords: Strategies of fortification, coastal villas, church-bastions.

\section{Introducción: los antecedentes medievales}

A mediados del siglo XIII la conquista cristiana, del Reino de Valencia convirtió su espacio marítimo en un territorio de frontera con el Islam. Frontera que fue percibida también como interna respecto de la mayoritaria población musulmana del interior del país. En el accidentado relieve del territorio de la Marina de la costa alicantina el dominio feudal fue posible mediante la ocupación selectiva de lugares estratégicos. Los establecimientos de la colonización feudal valoraron cuidadosamente sus especificidades geográficas y ocuparon aquellos asentamientos andalusíes que permitían asegurar tanto el dominio efectivo del territorio litoral - la frontera exterior - como el control sobre las vías de penetración al accidentado interior del país - la frontera interior- De este modo, en la Marina Alta, después de la caída de la urbe de Denia, los nuevos pobladores llegados en 1258 afianzaron el cierre del litoral, con la ocupación del castillo de Calpe, las alquerías de
Benissa, Teulada, Jávea y Pego. La colonia de Murla, el único asentamiento tierra adentro, ejercía el control de la salida de los valles interiores a la principal vía de comunicación de la costa. Algunos de estos asentamientos, como los de Benissa y Pego, incluso asumieron ambas funciones. Sin embargo un exclusivo punto de vista de la configuración y defensa de esta frontera sería insuficiente para explicar por sí sólo este proceso que debía de garantizar la renta feudal con la continuidad de la explotación de los recursos agrarios. Sería, pues, la estrategia de dominio y continuidad de la estructura funcional y productiva del territorio la que, junto al control y defensa de la frontera litoral, garantizaría precisamente el éxito y permanencia de las nuevas villas de colonización feudal. En ello reside el continuado fracaso de las reiteradas tentativas de fundación y establecimiento de una villa de nueva planta en el peñón de Ifac en 1282, 1298 y 1340, destinada a trasladar a todos 
los nuevos pobladores de las colonias del territorio jurisdiccional del castillo de Calpe, esto es, los pobladores ya establecidos en 1258 junto al propio castillo y en las antiguas alquerías de Benissa y Teulada. La villa de nueva fundación en las faldas de Ifac era, sin duda, adecuada y conveniente para los intereses de la Corona desde el punto de vista de la estrategia de defensa costera pero ineficaz para vertebrar las funciones y estructura territorial asentadas desde siglos en este ámbito geográfico. El saqueo y destrucción de la villa de Ifac en 1359 por una escuadra de naves genovesas y castellanas, fue el acontecimiento que precipitó definitivamente la construcción de la villa medieval de Calpe y, como en los otros casos, en torno a la fortificación de la primitiva alquería musulmana (Banyuls y Crespo, 2014).
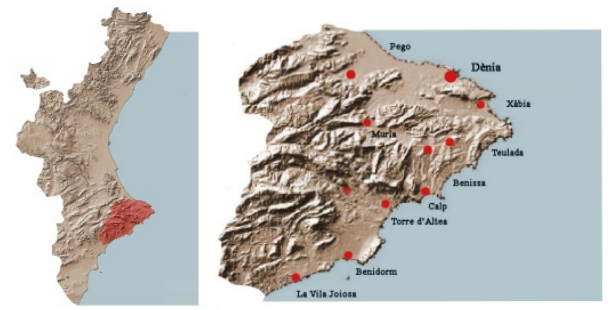

Fig. 1- Ámbito territorial de la Marina. Relieve y asentamientos urbanos ss. XIII-XIV.

De esta forma, en la época medieval se había configurado un sistema de villas en la Marina Alta surgidas al resguardo de unas estructuras defensivas preexistentes. En la mayoría de los casos los colonos se valieron de las torres de defensa y reductos de las alquerías andalusíes ocupadas. Así los asentamientos de Calpe -también el primigenio efectuado al resguardo del castillo- Benissa, Teulada, Jávea y Murla surgieron inicialmente al pie de una estructura militar elemental andalusí que constituyó el punto de partida del desarrollo urbano.

En estas villas las iglesias como estructura colectiva básica donde se desenvolvían además de las funciones religiosas también otras civiles, eran utilizadas, a su vez, como dispositivo de fortificación para refugio y defensa de la población. En casos, como Benissa y Teulada, sus iglesias se construyeron reutilizando en parte la estructura defensiva existente, en otros, como
Calpe, Jávea, Pego o Murla, se edificó como estructura de defensa independiente a la torre andalusí y en el caso de la fracasada bastida de Ifac como fortaleza única. En todos los casos, y debido a este cometido también de defensa, se emplazaron estratégicamente en uno de los vértices expuestos de su recinto amurallado. Las villas medievales de Benissa y Pego también respondieron originariamente a este esquema básico aunque tempranamente con la llegada de nuevos pobladores tuvo que ser modificado. El recinto amurallado y estructura urbana medieval de Pego y Benissa es en realidad el resultado de dos fases sucesivas de urbanización reconocibles aún en la topografía urbana.

\section{Forma y estrategias de fortificación en la primera etapa de modernización (1535-1563)}

La función estratégica de cierre y defensa del litoral valenciano que ejercieron estas villas desde época medieval cobraría una especial relevancia a finales del siglo $\mathrm{XV}$, cuando el territorio de la Marina adquiría la condición de avanzado bastión en un Mediterráneo convertido en campo de fuerzas de las nacientes potencias imperiales. La escalada de las tensiones se dejó sentir con especial intensidad durante el primer tercio del siglo XVI. En 1518 fue asaltada la ciudad de Denia, en 1529 una armada argelina saqueó la villa de Murla y destruyó su iglesia. En 1531 y 1535 la villa de Benissa recibió dos asaltos de consecuencias mortales para pobladores y asaltantes. En este último suceso se constataba, además, la obsolescencia de las murallas medievales y la problemática añadida del crecimiento urbano fuera del recinto medieval.

El continuo estado de guerra puso de manifiesto la precaria situación en que se encontraban las defensas medievales las cuales -cabe señalarse- desde hacía más de un siglo no habían tenido ninguna necesidad de modificarse. Ahora las innovaciones técnicas introducidas en la guerra, con el uso generalizado del armamento de fuego, repentinamente convertían en obsoletas estas antiguas defensas de manera que exigieron una adaptación muchas veces radical. 
De este modo el retumbar de la artillería se convirtió en un premonitorio signo de cambio a unos nuevos tiempos que introdujeron el arte del Renacimiento en este territorio y justamente desde la vertiente funcional y técnica de las obras de defensa. El Renacimiento, entendido como un proceso de racionalización, avance científico y consciente cambio de concepción respecto de un pasado medieval, entraba súbitamente en este territorio de la mano de la nueva arquitectura para la guerra.

A finales de la década de 1530 se exigió la puesta en marcha de obras para una necesaria adaptación de las antiguas estructuras de defensa medieval. Las primeras intervenciones consistieron en implementar nuevos dispositivos de fortificación sobre las estructuras medievales existentes. Generalmente se emplearon torres circulares de mayor amplitud y menor altura con bases terraplenadas concebidas para poder alojar piezas de artillería y resistir el impacto de los proyectiles sobre ellas.

Así entre 1530 y 1545 se edificaba la nueva iglesia-fortaleza de la villa de Murla (Giner, 1995) y se iniciaban obras en Calpe, Benissa y Teulada para reforzar las murallas, levantar nuevas torres y ejecuta $r$ nuevos muros de cierre de los arrabales (Banyuls, Boira y Lluesma, 1996). Así mismo las obras para dar continuidad a la iglesia de Jávea, reiniciadas con nueva traza en 1513 (Bérchez, 1994), la dotaban también de este carácter de fortaleza. Seria ahora la necesidad de desarrollo de este cometido de fortificación o baluarte el que cobraría protagonismo incluso por encima del de sus propias funciones religiosas: «que.s veu de cada dia quant és necessari que.s derroquen les yglesies si lo ediffici de aquelles ha de fer dany $y$ que les terraplenen per a fer-ne baluarts» ${ }^{1}$

La nueva fortaleza e iglesia de la villa de Murla representaba la consecución más palpable de estas nuevas circunstancias en la inversión conceptual producida al sustituir un tipo tradicional de iglesia con elementos añadidos para su fortificación por una nueva concepción tipológica de fortaleza que subsidiariamente adaptaba las funciones religiosas.
Para la fortificación de Calpe se proyectó doblar las murallas medievales, recreciéndolas con terraplenes y añadir dos nuevas torres artilladas en los vértices de levante y tramontana. En 1551 la edificación de la mayor de las dos torres -la situada a poniente y significativamente denominada como «la torre grossa»- estaba finalizada. Las obras de la situada en el vértice diagonalmente opuesto trataban de principiarse ese mismo año pero su efectividad exigía el derribo previo y conflictivo de algunas viviendas preexistentes tanto en el interior del recinto medieval como exteriormente en el arrabal: «per que la dita torre axí per part de dins com de fora stiga appartada de altres edefficis necessariàment se haura de demolir alguna part de casa eo cases del dit loch de Calp» ${ }^{2}$ Con las dos nuevas torres artilladas se perseguía finalmente un control visual y acción de tiro en todo el perímetro cuadrangular del recinto medieval. En 1551 también se había procedido a ya a engrosar con rellenos de tierra todo el lienzo sureste de la muralla medieval y se habían finalizado las obras de la torre y nuevo portal junto a la casa de la señoría.

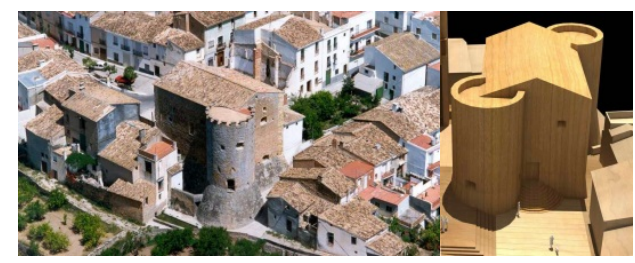

Fig. 2- Murla. Implantación urbana de la iglesiafortaleza y modelo volumétrico (A. Banyuls y S. Pastor, 2009)

En otros casos como el de la villa de Murla, donde adaptar la totalidad de su mayor pero también más extendido recinto medieval hubiese implicado una intervención de costes inasumibles, la alternativa adecuada fue la implantación de una fortaleza que permitiera puntualmente refugiar a toda la población. Ello se conseguía alojando el espacio para la iglesia en su interior. Esta fortaleza se proyectaba siguiendo los modelos de la moderna arquitectura militar que en aquellos momentos se había comenzado a gestar en las guerras de Italia, siendo referentes próximos las obras coetáneas de la alabada torre del Rey en Oropesa 
construida en 1535, el nuevo castillo de Oliva o la adaptación del castillo de San Martín en Jávea. A semejanza de éstas, la fortaleza de Murla se desarrollaba por un volumen rectangular de fuerte fábrica con torres o cubos redondos en dos de sus esquinas diagonalmente opuestas. En Murla el espacio interior se resolvió en dos niveles destinándose el inferior -cubierto con bóvedas de crucería- al espacio de la iglesia y el superior -cubierto con funcionales bóvedas de arista- al uso militar. La cubierta en terraza, como también se realizaba en la iglesia de Jávea, posibilitaba una plataforma para operar con artillería. Las dos torres angulares, provistas de troneras, eliminaban los ángulos muertos y permitían el alcance ofensivo sobre los cuatro flancos del edificio, actuando, además, sobre las enfiladas visuales de las principales calles y portales de la villa.

De manera similar en Benissa, que desde finales del siglo XV extendía su población fuera del cuadrilátero de la muralla medieval y cuya iglesia tempranamente ya había dejado de formar parte de la muralla del originario asentamiento feudal, la opción más adecuada participaba de las alternativas de fortificación vistas hasta ahora en las villas de Calpe y Murla, esto es, la inserción de nuevas torres circulares en el circuito de la muralla y la conversión de su iglesia como fortaleza artillada con capacidad de resguardar en su interior a la población.

La actuación en la antigua iglesia medieval consistió en el engrosamiento de sus muros y la edificación de dos nuevas torres circulares emplazadas flanqueando el ábside poligonal gótico. Una de estas torres, la situada al norte, en realidad embebía la primitiva torre-campanario que fue recortada en altura para igualarla, como la nueva, con la terraza del ábside. Estas dos torres como en la iglesia-fortaleza de la villa Murla, permitían controlar la enfilada de las dos calles principales y los portales de la población. La reestructuración de la iglesia se completó, además, con la ejecución de una barbacana perimetral al nivel de la cubierta en terraza de las capillas laterales ${ }^{3}$. De esta manera la reconversión de la iglesia gótica en fortaleza artillada posibilitaría la acción ofensiva en todos los frentes de la misma.

A pesar de ello, su emplazamiento, ocupando prácticamente una posición central en la estructura urbana de la villa, añadía una nueva problemática debido a la proximidad de las viviendas y la incompatibilidad que ello suponía con su función de fortaleza artillada. El mayor obstáculo, sin embargo, fue el que presentaba la situación de la casa abadía. Su ubicación aislada y su posición elevada frente a la escasa altura de la iglesia medieval la convertían en el principal reparo de la fortaleza: «com estant com esta la seglesia de Beniça baixa e la badia que hi esta al costat alta de aquella badia porien los enemichs ynvadir als que starien en la dita sglesia e aquella dita seglesia e fortalea no valdria res per ferse forts» ${ }^{4}$, por lo que, a pesar de la significación del edificio, se resolvió con su derribo.

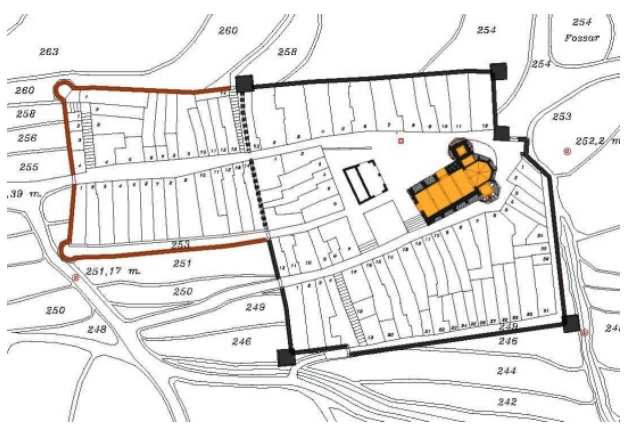

Fig. 3- Benissa. Estructura urbana y murallas ca. 1540 (A. Banyuls, 2009).

La estrategia adoptada en la villa de Teulada para adecuar las antiguas defensas medievales a las nuevas formas de fortificar consistió también en concentrar las escasas posibilidades de actuación sobre estructuras que permitiesen configurar una fortaleza capaz de albergar a la población, como alternativa a abordar unas gravosas obras de adecuación en todo el perímetro de sus murallas. En este caso la solución de fortificar la iglesia medieval pasaba también por fortificar un primitivo recinto o albacar en el interior del cual, y desde finales del s. XIII, se encontraba edificada. Este primitivo reducto -probablemente una adaptación en tiempos fundacionales del albacar andalusí- se 
situaba en el área más elevada de la villa y venía utilizándose como refugio de la población desde tiempos de la colonización feudal. Este antiguo reducto y la iglesia contenida en el mismo, siguiendo el esquema urbano básico que hemos descrito, también ocupaba, como bastión, uno de los vértices -el más elevado- en el circuito amurallado de la población (Ivars y Ivars, 1988).
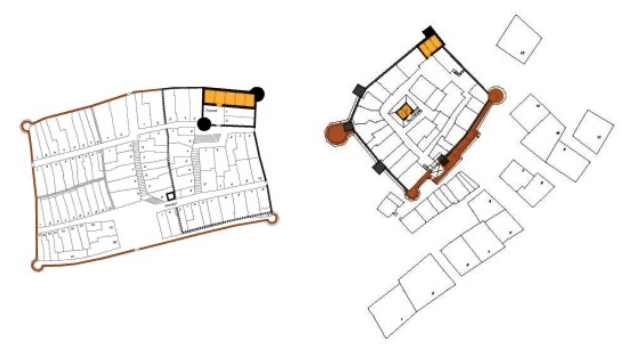

Fig. 4- Teulada y Calpe. Estructura urbana y murallas ca. 1560 (A. Banyuls, 2009).

La intervención llevada a cabo también fue la de inserir en el primitivo recinto cuadrangular dos nuevas torres circulares en dos de sus esquinas opuestas. Una de ellas, junto al ábside de la iglesia, ocupaba, a su vez, el vértice exterior del recinto amurallado de la villa y sugiere, como en Benissa, ser consecuencia del terraplenado y envoltura circular de una anterior torre medieval situada en el vértice del reducto y de la muralla de la población y probablemente con función de torre-campanario a la manera de las villas de Benissa e Ifac. La otra torre circular, construida de nueva planta $-y$ cuyas obras se remataban en 1551- se situaba en el vértice opuesto orientado hacia al interior de la población. De este modo las dos torres permitían defender con artillería la totalidad del perímetro exterior del recinto y, a su vez, dos lienzos de las muralla de la villa y uno de sus portales. Sin embargo, como sucedía también en Benissa, esta efectividad pasaba por la necesidad de derribar algunas viviendas que permitieran el aislamiento de la nueva fortificación así concebida.

La adecuación a las técnicas de la guerra moderna exigía a menudo de radicales intervenciones de demolición de las edificaciones preexistentes tanto para poder emplazar y operar los nuevos dispositivos como para eliminar los posibles reparos de los asaltantes. De este modo, los derribos, tanto o más que la edificación, constituyó uno de los instrumentos más eficaces de intervención y adaptación urbana a las nuevas necesidades de la defensa: «per que nunca bé se ha pogut ni.s pot fortificar alguna terra sense derrocar moltes coses que occorren, e moltes vegades la major fortificació és lo derrocar» ${ }^{5}$.

\section{La fortificación de Calpe entre 1562 y 1587: del proyecto del territorio a la introducción del baluarte. La visión moderna de los ingenieros militares}

En 1561, cuando la monarquía hispánica había asumido la necesidad de articular una estrategia global para defensa de los reinos peninsulares y ante la preocupación de una temida y probable invasión desde el norte de África, comisionó al ingeniero italiano Juan Bautista Antonelli para examinar el estado del litoral valenciano y determinar sus necesidades defensivas. En el informe que sobre la fortificación del reino valenciano elaboró el ingeniero real en $1563^{6}$ surgía una nueva y moderna visión renacentista en cuanto a la teorización, alcance y articulación territorial de las intervenciones propuestas. Para el ingeniero italiano la fortificación del territorio litoral debía regirse por los mismos principios que la fortificación una ciudad donde las villas más estratégicas con sus puertos serian las puertas de entrada, las demás villas costeras los baluartes y las torres de vigilancia y defensa costera, por último, serian como las almenas y garitas de la muralla del territorio en esa metáfora como muralla de la ciudad.

Sin embargo la mayor parte de las propuestas del proyecto ideal de J.B. Antonelli para el litoral valenciano, determinadas en dicho informe, no llegarían a materializarse en prescindir de las posibilidades económicas reales -pero también sociales- de los pueblos e instituciones valencianas sobre los cuales además debía recaer su financiación: «e conforme a la traça feta per lo dit Joan Baptiste Antonelli fonch manat a les dites ciutats, viles y lochs, se fortificassen his fessen les obres en dita traça contengudes, la 
qual fortificació sis fes seria total destructio e ruyna de dit regne» (Requena, 1997).

Este fue el caso de Calpe, donde J.B. Antonelli, ponderando sólo los intereses militares de la Corona $-\mathrm{y}$ con la misma errónea apreciación cometida en época medieval- estimó que era más conveniente efectuar una nueva implantación de la villa con moderna fortificación en el peñón de Ifac -que llegó a proyectar- que la fortificación en su emplazamiento y por ello debía de ser derribada en su totalidad. Sin embargo en el memorial anterior, elaborado después de su primer viaje en 1561 , con un contenido de mayor inmediatez sin reflexión teórica, la propuesta había sido justo la contraria, habiéndose incluso cuantificado económicamente las obras necesarias para la adecuada fortificación de la villa. De las villas de Benissa y Teulada, más retiradas del mar, J.B: Antonelli sólo recomendó, y en el primer memorial, realizar un reconocimiento para valorar si era más conveniente fortificar el perímetro de sus murallas o continuar con las actuaciones referidas, y aún en ejecución, de la fortificación de sus iglesias. Alternativas ambas que a la postre se llevarían a término.

Después del rechazo frontal al proyecto global de J.B. Antonelli, los esfuerzos económicos para la defensa del Reino se centraron puntualmente en fortalecer algunos de los lugares más expuestos y relevantes en la estricta línea marítima. En Calpe las obras de fortificación -que ahora: «tant convenen al servey de sa Magestad»- lograrían tener continuidad impulsadas por su inclusión como "baluarte" en la nueva dimensión adquirida por el territorio litoral del reino como muralla de ciudad. Sin embargo las obras para continuar con la renovación del recinto amurallado y adaptarlo a las nuevas exigencias técnicas en continua evolución conllevarían un largo tiempo de ejecución, no exento de interrupciones, que se prolongaría hasta 1587. Las nuevas necesidades de fortificación demandarían afectar, además, a algunas de las obras ejecutadas en la etapa anterior, como la torre artillada denominada de tramontana -en realidad, de orientación noreste- debido a la rápida obsolescencia de este tipo de torres para desplegar una función ofensiva de mayor alcance.

En una primera fase -desarrollada entre 1563 y 1575- se procedió a ensanchar y revestir este lienzo noreste de muralla y con ello la intervención sobre la iglesia, situada en el vértice norte del recinto amurallado, para su reconversión como nuevo baluarte artillado. Las obras de fortificación de la iglesia medieval consistieron, en este caso, en la ampliación de su antigua nave mediante la prolongación de un nuevo ábside y la extensión lateral con una nueva nave. Esta ampliación, ejecutada con gruesos muros en resalte al exterior a la muralla, junto a la sustitución de su originaria cubierta, muy probablemente de madera, por resistentes bóvedas permitía reforzar el ángulo de la muralla y generar una amplia plataforma superior para operar con artillería de mayor calibre. A su vez, su trazado poligonal impedía la creación de un ángulo muerto a las trayectorias de tiro rasante desde las contiguas torres circulares. Con todo ello se introducía en Calpe el primer ensayo de arquitectura abaluartada que vendría a sustituir los anteriores tipos de torres o cubos circulares artillados de base ataludada.

Sin embargo, y como se constata en la iglesiafortaleza de Murla, la introducción de innovaciones arquitectónicas en la articulación del espacio interior no había avanzado en la misma correspondencia que lo hacían las estructuras de fortificación se continuaba con el desarrollo de una tardía y tradicional concepción gótica de arcos apuntados, bóvedas de crucería y molduras medievales. No obstante en el nuevo ábside-baluarte de la iglesia de Calpe se introducían, contrariamente a la total articulación medieval de la iglesia de Murla, algunos tímidos motivos clasicistas como las aisladas columnas angulares de un particular orden toscano en las que descansan los arranques de las nervaduras góticas. Innovación introducida también con la edificación, entre 1562 y 1571, de la iglesia alicantina de Castalla y cuya cronología coetánea a la de las obras del baluarte de la iglesia de Calpe indica la participación de maestros que junto a las últimas 
novedades en la fortificación también tuvieron acceso a las del nuevo léxico arquitectónico a la romana.

Después de un enfriamiento de las inversiones en la costa alicantina entre 1575 y 1578, durante el virreinato de Vespasiano Gonzaga, las obras para abordar definitivamente la total renovación del encintado medieval se reiniciaron en 1579. En esta etapa además se introduciría las modernas formas y técnicas del sistema abaluartado que J.B. Antonelli había inaugurado en el territorio de la Marina con la edificación de la fortaleza de Bernia en 1562.

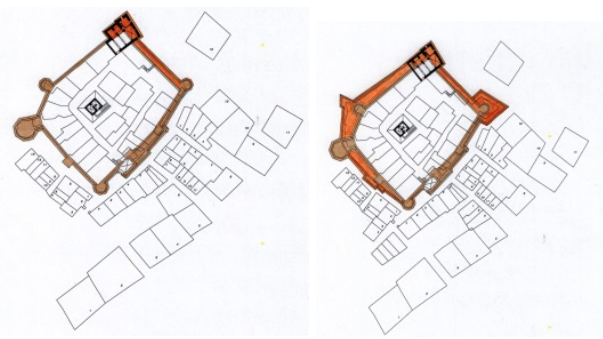

Fig. 5- Calpe. Edificación de las nuevas murallas y baluartes entre 1563-1575 (izquierda) y 1579-1587 (derecha) (A. Banyuls, 2009).

En mayo de 1579 el consejo de la villa contrataba los capítulos para las obras de reedificación de los lienzos de la muralla de poniente y mediodía todavía pendientes de realizar. Como en las anteriores actuaciones las obras consistían en un nuevo revestimiento y terraplenado de los antiguos muros medievales. La intervención contemplaba inicialmente aumentar la capacidad ofensiva del recinto con la ejecución de un nuevo baluarte conforme a su nuevo cometido de plaza fuerte: «congregat consell se fonch determinat doblar la muralla que ans tenien, redificantne una nova iunt a la antiga, y edificar un baluart pera posar sobre aquell una pesa de artilleria y tancar lo arrabal de dita vila» ${ }^{7}$.

Sin embargo, este baluarte no figuró en el acto de capitulación de las obras contratadas en 1579, probablemente por falta de la financiación suficiente, y todavía en 1581 no se había podido ejecutar: «que quant se determinà de fer en la dita vila de Calp y per los vehins de aquella dita muralla, se determina per lo semblant de fer lo dit baluart pa posar en aquell una peça $d^{\prime}$ artilleria, lo qual fins huy no es stat fet $\rangle^{8}$.

En la visita de reconocimiento y relación que en 1585 hizo Juan de Acuña sobre el litoral valenciano $^{9}$ Calpe aún poseía tres piezas de artillería y sólo dos de ellas - ya enumeradas en el informe del virrey Vespasiano Gonzaga de 1575- eran piezas de gran calibre. Estas se correspondían con las emplazadas en la «torre grossa» y en el baluarte de la iglesia. La tercera pieza de menor potencia era la ubicada en la más reducida torre del lienzo de tramontana.

No obstante las obras del nuevo baluarte -el cual significativamente vino a denominarse «de la peça»- se debieron iniciaron ese mismo año de 1585 o en el siguiente y con el impulso dado a las obras de fortificación en la costa del poniente valenciano auspiciado por el virrey Francisco de Moncada, marqués de Aytona (1580-1595). En 1587, y cuando Calpe ya operaba con cinco piezas gruesas de artillería, estas ya se encontraban finalizadas. La traza de este nuevo baluarte la atribuimos al ingeniero real Cristóbal Antonelli, sobrino y discípulo de Juan Bautista, que desde 1583 estuvo al cargo de las obras de fortificación de la costa valenciana. En 1585 también realizaba las trazas de los dos nuevos baluartes de la muralla de tierra de Benidorm y entre 1585 y 1595 se encargaba de las fortificaciones y nueva villa de Altea y de los proyectos para las torres del puerto de Moraira. Estas últimas obras en Altea y Moraira, junto a las del nuevo baluarte de Calpe, se situaban, además, y no casualmente, en la costa del territorio de la baronía de Calpe, señorío perteneciente al noble Francisco de Palafox que en 1590 se convertía en yerno del virrey.

El baluarte debía reforzar uno de los vértices más expuestos del renovado circuito de la ciudadela que posibilitara una acción ofensiva de largo alcance sobre la ensenada de Calpe con la dotación de piezas grandes de artillería. Este era el vértice orientado a levante donde se emplazaba la referida torre menor del lienzo de tramontana la cual, a pesar de su reciente construcción a mediados de la centuria, había quedado totalmente obsoleta con la rápida evolución de los modernos dispositivos de 
fortificación y las nuevas exigencias ofensivas. La torre fue cercenada y absorbida en la obra de terraplenado del nuevo baluarte. Con el nuevo baluarte se introdujo en Calpe definitivamente los nuevos diseños de fortificación renacentista de baluartes pentagonales mejor adaptados a las operaciones y trayectorias del tiro rasante de grandes piezas de artillería y más resistentes a sus efectos.

La villa de Calpe adquiría, de este modo, en 1587 una nueva condición de plaza fuerte con un cometido de vasto alcance como custodia y llave del reino que a la postre le otorgaba autoridad y prestigio territorial respecto de las otras villas de la baronía. Circunstancia que se manifestaba también en la moderna actitud adoptada de exaltación de sus potencialidades urbanas: «es més preeminent que los demes llochs de dita Baronia...per que en dita vila de Calp acostuma de haver y ha peces de artilleria y al present se troben cinch peces groses de artilleria, ab les quals, cuant los moros de allende venen y la terra no esta segura o desembarquen aquells, donen avis a tota la dita Baronia tirant dites peces $a b$ un home y a tota la costa y als demes llochs circunvehins a dita Baronia, lo que no poden fer los vehins y habitadorss de dit lloch de Benisa y Teulada» ${ }^{10}$.

A partir de esos momentos los enfrentamientos y luchas de poder por la hegemonía territorial con la villa de Benissa, que por otra parte fundaba su pujanza en su mayor población, extensión territorial, prestigio económico y antigüedad de fundación, estaban servidos.

\section{Notas}

(1) Archivo Real de Valencia. Real Audiencia, procesos de Madrid, exp. 83, letra J, f. 83r.

(2) Ídem, f. 86r.

(3) Ídem, f. 125r.

(4) Ídem, f. 125v.

(5) Ídem, f. 80v.

(6) Archivo General de Simancas. Estado, 329-I. Un análisis del informe en Boira J.V. (1992).

(7) Archivo Real de Valencia. Real Audiencia, procesos parte $2^{\circ}$, letra $S$, exp. 481, f. 2r. Transcrito en Llopis V. (1953).

(8) Ídem, f. 4v.

(9) Archivo de la Corona de Aragón. Consejo de Aragón, leg. 0761, nº 103, f. 10 v.

(10) Archivo Real de Valencia. Real Audiencia, procesos parte $2^{\circ}$, letra S, exp. 495, f. 21v. Proceso de la villa Calpe contra la villa de Benissa. Declaración del síndico de Calpe en abril de 1587. Sobre esta disputa territorial véase también Pastor J. (1986).

\section{Referencias}

Banyuls A., Boira J.V., Lluesma A. (1996). Arquitectura i control del territori: la defensa del litoral de la marina Alta al segle XVI. Institut de Cultura Juan Gil-Albert Ed. Alacant. pp. 34-37.

Banyuls A., Crespo T. (2014). "El poblament musulmà a l'antic terme del Castell de Calp (II). Estructura del paisatge i patrons d'organització territorial” en Mudejars, moriscs i cristians a la Marina y la Safor. 400 anys de l'expulsió. Institut Alacantí de Cultura Juan Gil-Albert Ed. Alicante.

Boira J.V. (1992). "Geografia i control del territori. El coneixement i la defensa del litoral valencià al segle XVI: l'informe de l'enginyer Joan Baptista Antonelli” en Cuadernos de Geografía $n^{\circ} 52$. Universitat de València Ed. Valencia. pp. 183-199.

Bérchez, J. (1994). Arquitectura·Renaixentista Valenciana (1500-1570). Bancaixa Ed. Valencia. p.52.

Giner S. (1995). Historia de Murla. Instituto de Cultura Juan Gil-Albert Ed. Alicante. p. 203.

Ivars J, Ivars J. (1988) “La vila de Teulada. Procés de fortificació y estructura urbana” en Aguaits $n^{\circ} 1$. Institut d'Estudis Comarcals de la Marina Alta Ed. Denia. pp. 49-64.

Llopis V. (1953) Calpe. Imprenta J. Nacher Ed. Valencia. p. 76

Pastor J. (1986) Les guardes i la capitalitat. Ajuntament de Benissa Ed. Benisa.

Requena F. (1997). La defensa de las costas valencianas en la época de los Austrias. Conselleria de Cultura, Educació i Ciència -Institut de Cultura Juan Gil-Albert Eds.Alicante. p. 11 